Case Reports

\title{
An Unusual Case of Acute Abdomen: Intestinal Ascariasis: Case Report and Literature Review
}

\author{
Ashaur Azhar and Rebecca Ann Lillis \\ Department of Medicine, Section of Infectious Diseases, \\ Louisiana State University Health Sciences Center, School of Medicine, New Orleans, Louisiana, USA
}

\author{
Article history \\ Received: 29-09-2019 \\ Revised: 8-11-2019 \\ Accepted: 12-11-2019 \\ Corresponding Author: \\ Ashaur Azhar \\ Department of Medicine, \\ Section of Infectious Diseases, \\ Louisiana State University \\ Health Sciences Center, School \\ of Medicine, New Orleans, \\ Louisiana, USA \\ Tel: $+1504-568-5031$ \\ Fax: +1504-568-5553 \\ Email: aazha1@1suhsc.edu
}

\begin{abstract}
Ascariasis is uncommon in United States of America but can be seen in immigrants and travelers. It can present with intestinal complications. We present a case of acute suppurative appendicitis with bowel perforation. Interestingly and unexpected, worms were palpated in the intestine in the operating room. Later, an adult Ascaris worm was identified in nasogastric tube canister. During hospitalization, a contrastbased computed tomographic scan revealed impressive finding of contrast filling defects in stomach and small intestine of the patient indicating the adult worms. The patient was treated with Ivermectin.
\end{abstract}

Keywords: Ascaris, Ascariasis, Round Worm, Nematode, Acute Appendicitis

\section{Introduction}

The genus Ascaris is derived from the Greek word askaris that means worm, that was first described by Linnaeus in 1758 (Khuroo, 1996). Ascaris lumbricoides is the largest intestinal nematode and the most common soil- transmitted human helminthic infection (Khuroo, 1996). It is not commonly seen in the United States (US) but can be seen in immigrants and travelers to this area. People can get infected with soil, water and children's toys contaminated with fertile eggs. Even transplacental transmission has been reported (Chu et al., 1972). The eggs can survive for up to 10 years in favorable climate (moist, warm and shaded soil) (Khuroo, 1996). Ascariasis is most often asymptomatic. Symptomatic infections can have pulmonary manifestations in the early larval stage and can have several intestinal (intestinal obstruction most commonly at ileocecal valve, appendicitis, gangrene, volvulus, intestinal perforation) and biliary/pancreatic (biliary colic, cholecystitis, liver abscess, pancreatitis) manifestations in the late adult worm stage. They can present as malnutrition with vitamin A and C deficiency (Taren et al., 1987). Vitamin deficiencies may lead to growth and intellectual compromise in infected children (Bethony et al., 2006). We present a case of acute suppurative appendicitis with bowel perforation, later determined to be secondary to Ascaris worm infestation.

\section{Case Report}

A 36-year-old male immigrant from Guatemala presented to our Emergency Department (ED) with a 3day history of fever, abdominal pain, nausea and emesis. Physical examination showed a malnourished and toxic appearing patient with an acute abdomen with diffuse abdominal cramping. Laboratory testing revealed normal white cell count with only elevated neutrophils and bands, hypokalemia, lactic acidosis and acute kidney injury with creatinine level of $4.08 \mathrm{mg} / \mathrm{dl}$ (normal: $0.7-$ $1.4 \mathrm{mg} / \mathrm{dl}$ ). Non-contrasted Computed Tomographic (CT) scan of abdomen demonstrated acute suppurative appendicitis with perforation and abscess. The patient underwent appendectomy during which worms were palpated in the mid-jejunum in the operating room. Two days later, an adult Ascaris worm was identified in nasogastric tube canister (Fig. 1). After few days when renal function improved a subsequent CT scan with contrast revealed filling defects in stomach and small intestine indicating the adult worms in the axial and cross-sectional view (Fig. 2 and 3). After patient's bowel function returned, he was treated with Ivermectin. 

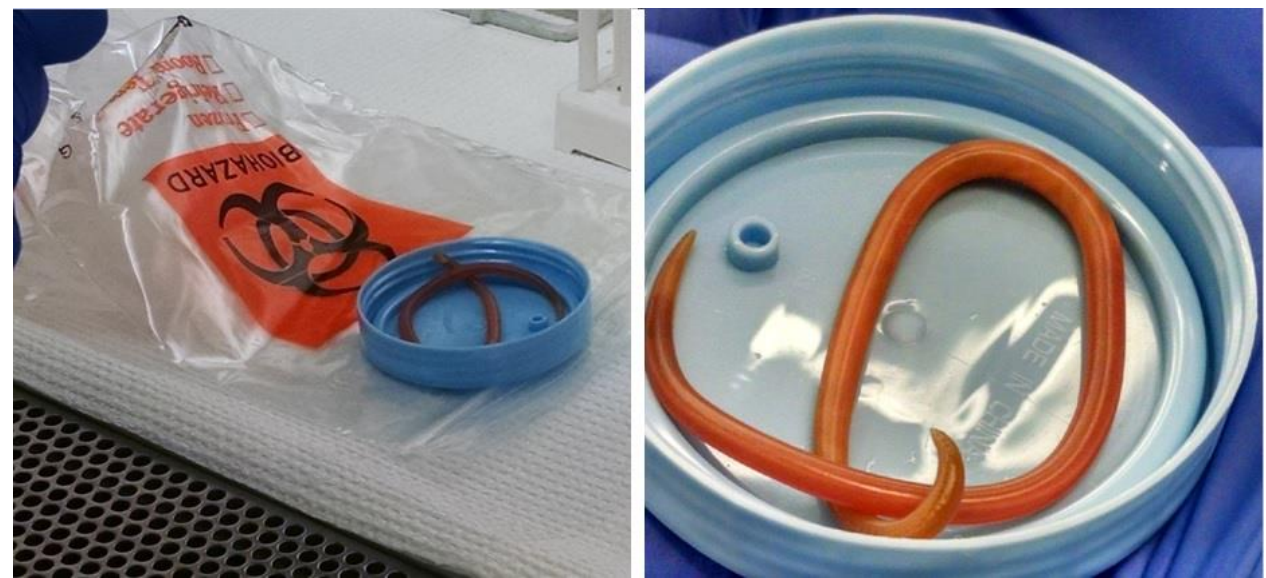

Fig. 1: Photograph of the Ascaris adult worm from a distance (left) and a close up (right) in the microbiology laboratory from the patient's nasogastric tube canister

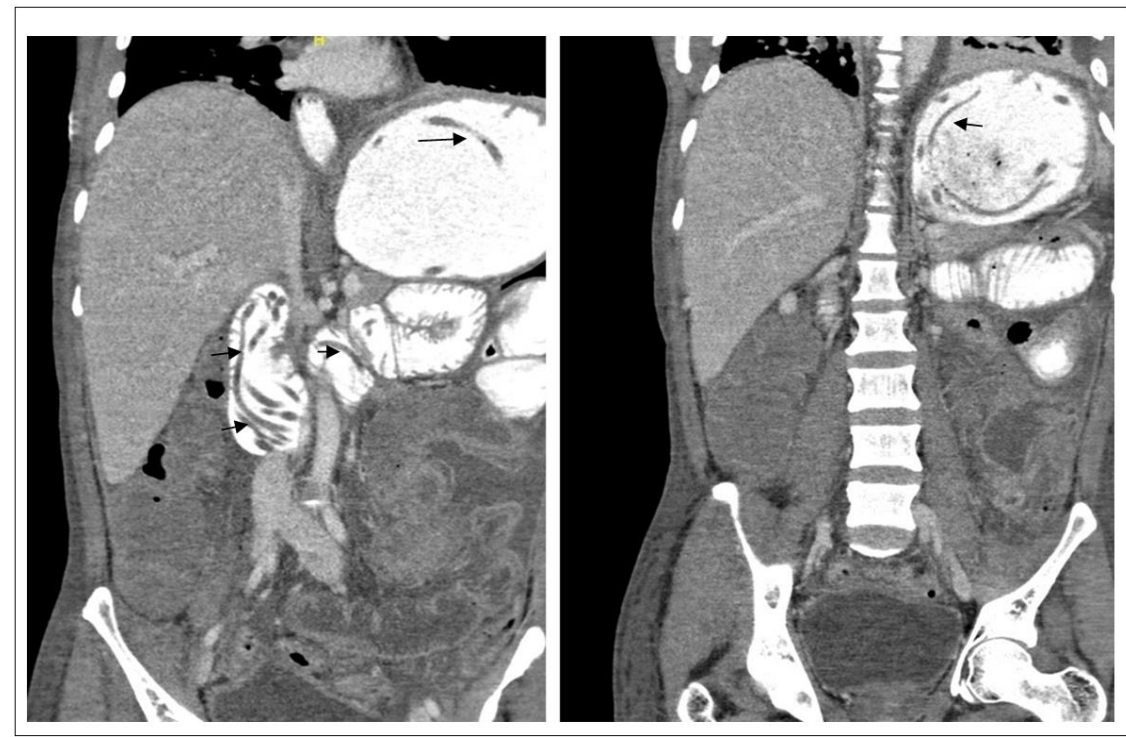

Fig. 2: CT scan with contrast revealing filling defects in stomach and small intestine indicating the adult worms in the axial views

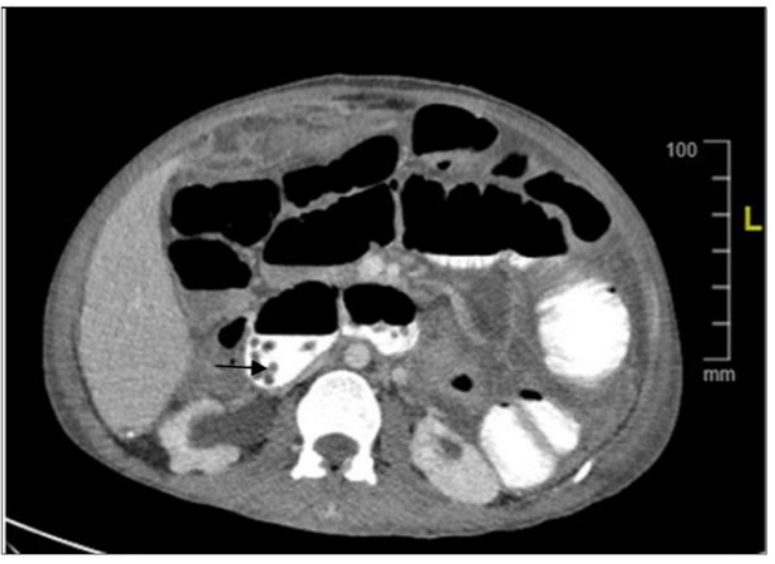

Fig. 3: CT scan with contrast revealing filling defects in intestine indicating the adult worms in a cross-sectional view

\section{Discussion}

An estimated 807 million-1.4 billion people in the world are infected with Ascaris lumbricoides (Khuroo, 1996; Bethony et al., 2006). Most individuals with ascariasis live in Asia (73\%), Africa (12\%) and South America (8\%) (Pullan et al., 2014). Historically, the highest burden of ascariasis in the U.S. occurred in the southeast region; the prevalence of infection has decreased with better sanitation (Starr and Montgomery, 2011). Of the 4 million people infected in the United States, a large percentage are immigrants with infection rates of $20 \%$ to $60 \%$ as per World Health Organization (WHO) report (WHO Geneva, 1981).

Mature adult female worms can measure 20 to $49 \mathrm{~cm}$; males 15 to $30 \mathrm{~cm}$ (Khuroo, 1996). There are some secretory molecules known to be linked with Ascaris 
suum (a close relative of Ascaris lumbricoides, that infects swine) that may play a role in protective mechanism of these worms in the host body. According to some authors ( $\mathrm{Ng}$ et al., 2000), it is opined that the worms can secrete PI-3 (a pepsin inhibitor) that is thought to protect maturing worms from digestive enzymes in the stomach before they reach the small intestine. Other authors (Deehan et al., 2002) believe they can secrete Phosphorylcholine (PC) that is linked to glycoprotein glycans or glycolipids that can interfere with lymphocyte proliferation that can lead to suppression of lymphocyte responses in ascariasis. This also leads to inhibition of the production of some cytokines like interferon gamma by T-helper 2 cells.

\section{Diagnoses}

Diagnoses always start with a good history taking and thorough physical examination. Malnourishment and growth retardation can be evident in these patients especially children with impaired cognitive development. In laboratory data, eosinophilia is uncommon (seen mostly in early larval pulmonary phase but not in the adult wormintestinal phase). Low albumin level may be noted. As mentioned above, vitamin deficiencies can be present. Our patient also had low vitamin A and D serum levels. In microscopy, stool examinations for the eggs and parasites can be ordered. Stool concentration methods for detection of Ascaris eggs include Kato-Katz and FLOTAC techniques, the former technique being the preferred technique by WHO, but its sensitivity decreases with high intensity infection. FLOTAC is more sensitive than KatoKatz technique but its use is limited in situations where there is no centrifuge available. Polymerase Chain Reaction (PCR) is more sensitive and specific than microscopy but used more in epidemiological settings. Serology can have cross reactivity with other helminths' antigens (Reeder, 1998). PCR and Serology are not widely used in clinical diagnosis yet. It is believed that ascariasis produces detectable Immunoglobulin (Ig) G antibodies to Ascaris lumbricoides, but these IgG antibodies do not appear to have protection against infection (McSharry et al., 1999). But some authors (Gazzinelli-Guimarães et al., 2018) in Houston, Texas and Brazil, have proved that $\mathrm{IgG}$ induced by vaccination from extracts of Ascaris suum, has protection against ascariasis but in animal model though.

Imaging can play an important role in diagnosis (Reeder, 1998 and Pylant et al., 2006). Plain X-ray imaging of the abdomen can show a whirlpool effect. Ultrasonography has been used to identify Ascaris worms in pediatric patients. Ultrasounds can demonstrate intestinal echogenic tubular structures, curved strips, or a "target" sign. In children and adults, barium imaging of the small bowel can outline the individual worms as elongated radiolucent filling defects within the intestinal barium column. The worms may ingest barium; in such cases, the worm's alimentary canal appears as a white thread bisecting the length of the worm's body. Contrast based CT scans and Magnetic Resonance Images (MRI) of the bowels can show as filling defects or "Bull's eye appearance.

\section{Treatment}

For non-pregnant individuals, the benzimidazoles (Albendazole and Mebendazole) are considered the mainstay of treatment. Some authors (Belizario et al., 2003) have shown good therapeutic results with Ivermectin also, in comparison to Albendazole. In pregnant individuals, Pyrantel Pamoate is preferred since benzimidazoles have shown to be teratogenic in animals but as per Centers for Disease Control and Prevention (CDC) website, WHO allows use of Albendazole in the second and third trimesters of pregnancy in certain conditions like during mass prevention campaigns.

Our patient was treated with Ivermectin due to unavailability of benzimidazoles in the hospital at that time. Ivermectin dose for Ascariasis is 150 to 200 microgram (mcg) per Kilogram (kg) by mouth, one dose. We recommended $200 \mathrm{mcg} / \mathrm{kg}$ times one dose by mouth. The patient was given $12000 \mathrm{mcg}$ orally, once, since his documented weight at that time was $59 \mathrm{~kg}$. He stayed in the hospital for 9 more days for his postoperative recovery. Patient clinically improved and was discharged. He did not follow-up after discharge.

\section{Conclusion}

Ascariasis is not common in this area of the world, but clinicians and surgeons should be aware of it and should include it in the differential diagnoses of acute abdominal pain from acute appendicitis in immigrants and travelers. Transmission of infection is enhanced by asymptomatically infected individuals who can continue to shed eggs for years. That is why not only symptomatic but also asymptomatic individuals should be treated. Therapy should not be started if patient is constipated or obstructed. Prior infection may not confer protective immunity in humans infected with Ascaris lumbricoides. Infestations are ordinary not opportunistic, hence, thus usually show no correlation to immune status of the patients (Wiwanitkit, 2006). Measures should be taken to develop strategies for better sanitation, health education and a screening method for adult immigrants and refugees where the prevalence of soil-transmitted helminths is especially high.

\section{Acknowledgement}

We would like to thank the departments of radiology (for reviewing the $\mathrm{CT}$ scans with the corresponding author) and pathology/microbiology at 
University Medical Center, New Orleans, Louisiana, USA for their contribution.

\section{Author's Contributions}

Ashaur Azhar: Designed, wrote and reviewed the manuscript.

Rebecca Ann Lillis: Reviewed the manuscript and gave final approval.

Both authors were involved in the care of the patient in the hospital.

\section{Ethics}

This article is original and contains unpublished material. The corresponding author confirms that all the other authors have read and approved the manuscript and no ethical issues involved.

\section{References}

Belizario, V.Y., M.E. Amarillo, W.U. de Leon, A.E. de los Reyes and M.G. Bugayong et al., 2003. A comparison of the efficacy of single doses of albendazole, ivermectin and diethylcarbamazine alone or in combinations against Ascaris and Trichuris spp. Bull. World Health Organ., 81: 35-42 PMID: 12640474

Bethony, J., S. Brooker, M. Albonico, S.M. Geiger and A. Loukas et al., 2006. Soil-transmitted helminth infections: ascariasis, trichuriasis and hookworm. Lancet, 367: 1521-1532 DOI: $10.1016 / \mathrm{S} 0140-6736(06) 68653-4$

Chu, W.G., P.M. Chen, C.C. Huang and C.T. Hsu, 1972. Neonatal ascariasis. J. Pediatr., 81: 783-785. DOI: 10.1016/S0022-3476(72)80103-3

Deehan, M.R., H.S. Goodridge, D. Blair, G. Lochnit and R.D. Dennis et al., 2002. Immunomodulatory properties of Ascaris suum glycosphingolipids: phosphorylcholine and non-phosphorylcholinedependent effects. Parasite Immunol., 24: 463-469 DOI: 10.1046/j.1365-3024.2002.00489.x

Gazzinelli-Guimarães, A.C., P.H. Gazzinelli-Guimarães, D.S. Nogueira, F.M.S. Oliveira and F.S. Barbosa et al., 2018. IgG induced by vaccination with Ascaris suum extracts is protective against infection. Front Immunol., 9: 2535-2535.

DOI: $10.3389 /$ fimmu.2018.02535
Khuroo, M.S., 1996. Ascariasis. Gastroenterol. Clin. North Am., 25: 553-77. DOI: $10.1016 / \mathrm{S} 0889-8553(05) 70263-6$

McSharry, C., Y. Xia, C.V. Holland and M.W. Kennedy, 1999. Natural immunity to Ascaris lumbricoides associated with immunoglobulin $\mathrm{E}$ antibody to ABA-1 allergen and inflammation indicators in children. Infect. Immun., 67: 484-484. https://iai.asm.org/content/67/2/484.long

Ng, K.K., J.F. Petersen, M.M. Cherney, C. Garen and J.J. Zalatoris et al., 2000. Structural basis for the inhibition of porcine pepsin by Ascaris pepsin inhibitor-3. Nat. Struct. Biol., 7: 653-57. DOI: $10.1038 / 77950$

Pullan, R.L., J.L. Smith, R. Jasrasaria and S.J. Brooker, 2014. Global numbers of infection and disease burden of soil transmitted helminth infections in 2010. Parasit. Vectors, 7: 37-37. DOI: $10.1186 / 1756-3305-7-37$

Pylant, A., J.W. Hinshaw, R.B. Leonard and S. Zelman, 2006. Intestinal ascariasis: CT findings and diagnosis. South Med. J., 99: 317-8. DOI: $10.1097 / 01 . s m j .0000202702 .32649 .26$

Reeder, M.M., 1998. The radiological and ultrasound evaluation of ascariasis of the gastrointestinal, biliary and respiratory tracts. Semin. Roentgenol., 33: 57-78. DOI: 10.1016/S0037-198X(98)80031-X

Starr, M.C. and S.P. Montgomery, 2011. Soil-transmitted helminthiasis in the United States: A systematic review--1940-2010. Am. J. Trop. Med. Hyg., 85: 680-684. DOI: 10.4269/ajtmh.2011.11-0214

Taren, D.L., M.C. Nesheim, D.W. Crompton, C.V. Holland and I. Barbeau et al., 1987. Contributions of ascariasis to poor nutritional status in children from Chiriqui Province, Republic of Panama. Parasitology, 95: 603-613. DOI: $10.1017 / \mathrm{s} 0031182000058029$

WHO Geneva, 1981. WHO scientific group on intestinal protozoan and helminthic infections and World Health Organization. Intestinal protozoan and helminthicinfections: Report of a WHO scientific group Intestinal protozoan and helminthic infections: WHO Technical Report Series 666.

Wiwanitkit, V., 2006. Intestinal parasite infestation in HIV infected patients. Curr. HIV Res., 4: 87-96. DOI: $10.2174 / 157016206775197682$ 